\title{
A Short Survey Of Iris IMAges Databases
}

\author{
Mustafa M. Alrifaee, Mohammad M. Abdallah and Basem G. Al Okush \\ Al-Zaytoonah University of Jordan, Amman, Jordan
}

\begin{abstract}
Iris recognition is the most accurate form of biometric identification. The robustness of iris recognition comes from the unique characteristics of the human iris texture as it is stable over the human life, and the environmental effects cannot easily alter its shape. In most iris recognition systems, ideal image acquisition conditions are assumed. These conditions include a near infrared (NIR) light source to reveal the clear iris texture as well as look and stare constraints and close distance from the capturing device. However, recent advances on iris recognition have proposed different methods to process iris images captured in unconstrained environments. These environments include a visible wavelength (VW) light source, on the move and over distance from the capturing device. This research states the most used iris databases and describes their imaging framework along with all characteristics of iris images in each database.
\end{abstract}

\section{KEYWORDS}

Iris recognition, unconstrained environments, near infrared light, visible wavelength.

\section{INTRODUCTION}

In this paper, we will introduce the mostly cited human iris databases with their characteristics, which have been created for research purposes and experiments. All the databases that will be described in this paper are public and freely available on the internet. In the following subsections, the characteristics of eight different iris databases will be described. Then a comparison between all described databases will be stated and last section will address the current applications of iris recognition system.

\section{IRIS IMAGE DATABASE}

The biometric process encompasses an automated method of recognizing human individuals, based on their biological characteristics. In the past thirty years, the field of biometric recognition gained the interest of many researchers, and several proposals have been introduced. While these proposals need to be proved, a large number of tests over a large number of subjects must be performed. So, it is not realistic for researchers to collect their own dataset, due to its difficulties that this could imply. Also, for a robust comparison, different proposals should be implemented over the same dataset. Therefore, a benchmark database is necessary and cannot be sacrificed for the sake of recognition development.

The key behind any recognition system is the failure of a test of statistical independence [1] for the random textures offered by the human iris, the iris image involves so many degrees-offreedom (249) as a complex textures [2,3]. Many parameters in the iris recognition system should be considered, and the optimal threshold values to these parameters should be assigned to ensure the maximum recognition accuracy. These parameters include the dimension of input image, the radial and angular resolution of the normalized rectangular image $r$ and $\theta$ respectively. That will be used for the calculation of the number of coefficients extracted. Daugman's proposal 
[1], uses 2,048 bit iris template to calculate the hamming distance under different dissimilarity threshold values to calculate the recognition system accuracy.

As seen in Fig. 1. The false match rate of the processed iris template is reliable and have a potential for security in many fields.

\begin{tabular}{lc}
\hline HD criterion & Odds of false match \\
\hline 0.26 & 1 in $10^{13}$ \\
0.27 & 1 in $10^{12}$ \\
0.28 & 1 in $10^{11}$ \\
0.29 & 1 in 13 billion \\
0.30 & 1 in 1.5 billion \\
0.31 & 1 in 185 million \\
0.32 & 1 in 26 million \\
0.33 & 1 in 4 million \\
0.34 & 1 in 690,000 \\
0.35 & 1 in 133,000 \\
0.36 & 1 in 28,000 \\
0.37 & 1 in 6,750 \\
0.38 & 1 in 1,780 \\
0.39 & 1 in 520 \\
0.40 & 1 in 170 \\
\hline
\end{tabular}

Figure 1. Hamming Distance and Equivalent False Match [1]

The followings are the free available iris image databases:

\subsection{CASIA IRIS DATABASE}

It is the first freely available iris database for researchers, produced by the Chinese Academy of Science - Institute of Automation, will be addressed as CASIA onward in this document [4]. The first versions of CASIA iris databases utilize ideal acquisition environment, with stop and stare, at a close distance and use NIR (Near Infrared) light sources, which simulate the same conditions that have been proposed and used by Daugman's iris recognition system.

From the date of CASIA database creation, the Chinese Academy of Science produced four versions of CASIA databases as follows:

\subsubsection{CASIA-IRIS V1}

This version contains 756 iris images from 108 human subjects, each eye image has seven different classes divided into two sessions, training session-1 (3 images) and testing session-2 (4 images). The images in CASIA-Iris V1 have been captured using homemade iris camera, with eight circularly fitted NIR $850 \mathrm{mn}$ illuminators [4]. Before releasing the database to the public, and to compensate the effect of specular reflection, the area of the pupil is replaced with a constant intensity black circle. All the images are stored in format of bmp at $320 * 280$ resolutions. Figure 2 shows the capturing framework for CASIA database.

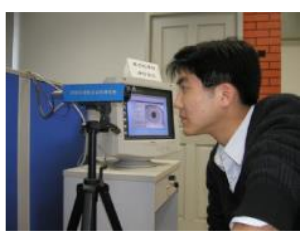

(a)

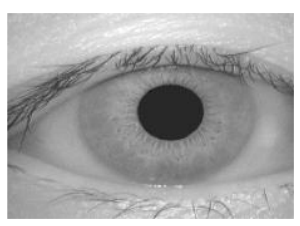

(b)

Figure2. CASIA-Iris V1 (a) capturing device, (b) captured image [4] 


\subsubsection{CASIA-IRIS V2}

This database contains 2400 images [4], divided into two subsets captured by two different devices. The first 1200 images were captured by Irispass-h device developed by OKI, and the other 1200 images were captured by CASIA-Iris cam developed at the Chinese Academy of Science.

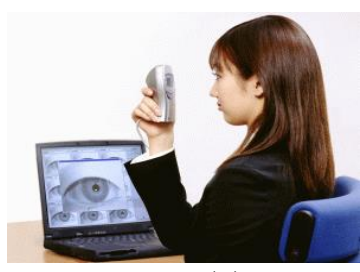

(a)

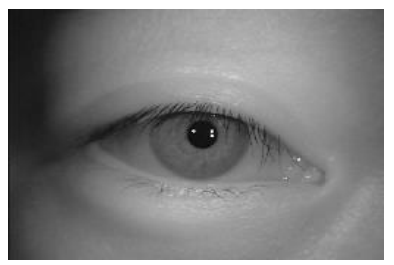

(b)

Figure 3. CASIA-Iris V2 Device 1 (a) imaging device (b) result image [4]

Each iris image has 60 different classes; all images are stored in bmp format at $640 * 480$ resolution. Figure 3 and Figure 4 shows the imaging devices used to capture the iris images in CASIA V2 database.

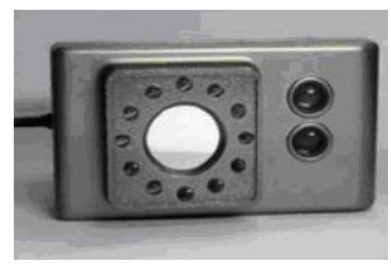

(a)

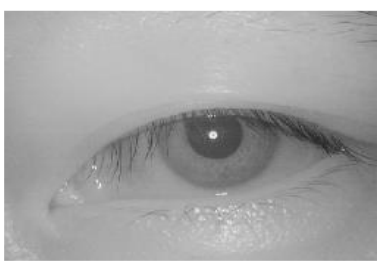

(b)

Figure4. CASIA-Iris V2 Device 2 (a) imaging device (b) result image [4]

\subsubsection{CASIA-IRIS V3}

This is the first CASIA database that introduced noise factors. It contains a total of 22,034 iris images collected from 700 humans. The database is divided into 3 subsets. The Interval data subset contains 2,639 images with very rich texture at a very close distance and lighted with an array of LED NIR light source, all images are stored in jpg format of 320*280 resolutions.

The Lamp subset contains 16,212 iris images with visible light on-off factor, which will cause the dilation in pupil size and a non-linear deformation in the captured images, images are stored in format of jpg and $640 * 480$ resolution. Finally, the twin's data subset which contains 3,183 images captured from 100 pairs of twins during the annual twin's festival in Beijing, all images are stored in jpg format at $640 * 480$ resolutions. Figure 5, Figure 6 and Figure 7 shows sample images from CASIA V3 Interval, Lamp and Twins respectively.

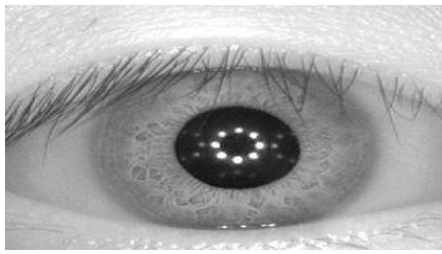

(a)

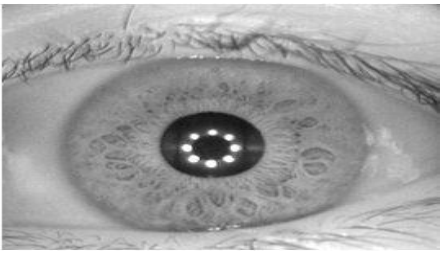

(b)

Figure5. Samples from CASIA-Iris V3 Interval [4] 
The International Journal of Multimedia \& Its Applications (IJMA) Vol.9, No.2, April 2017

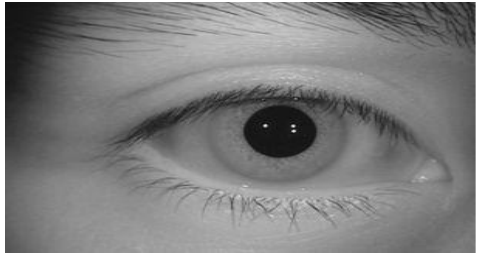

(a)

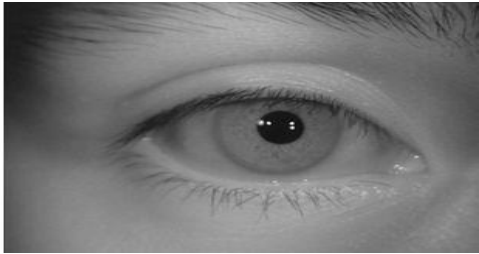

(b)

Figure6. Samples from CASIA-Iris V3 Lamp [4]

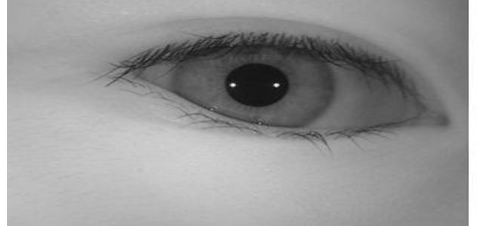

(a)

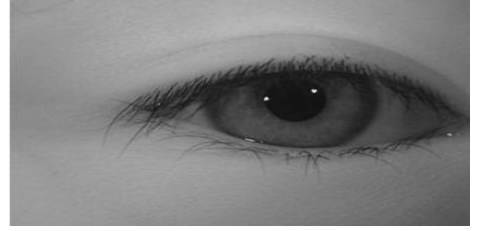

(b)

Figure 7. Samples from CASIA-Iris V3 Twins [4]

\subsubsection{CASIA-IRIS V4}

Early iris recognition systems deal with iris images captured with human subject constrains, while current research contributions are dedicated to relax these constrains [4]. Therefore, CASIA started working to produce a new iris images database which contains images captured on subject moving, over a distance and with poor quality under the name of CASIA-Iris V4.

CASIA-Iris V4 is an extension to CASIA-Iris V3 with three more subsets. The first one is called CASIA-Iris-Distance. In this dataset, the iris images have been captured over 3 meters' distance and while the subject is moving. The framework is shown in figure 8 taken from CASIA website [4], the database contains 2576 images with resolution of $2352 * 1728$.

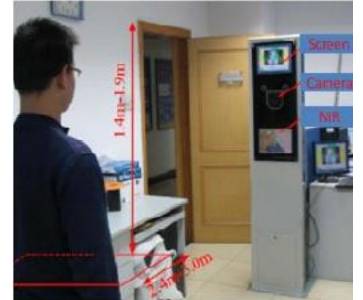

(a)

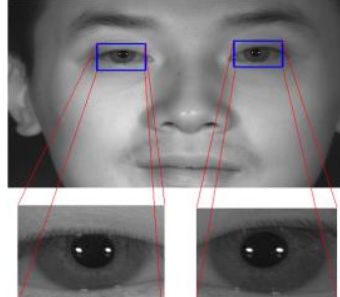

(b)

Figure 8. CASIA-Iris V4 Distance (a) imaging device (b) result image [4]

The second set is called CASIA-Iris-Thousand shown in figure 9. This dataset uses IKEMB-100 dual camera with friendly interface, and the output image reveals texture as "what you see is what you get"

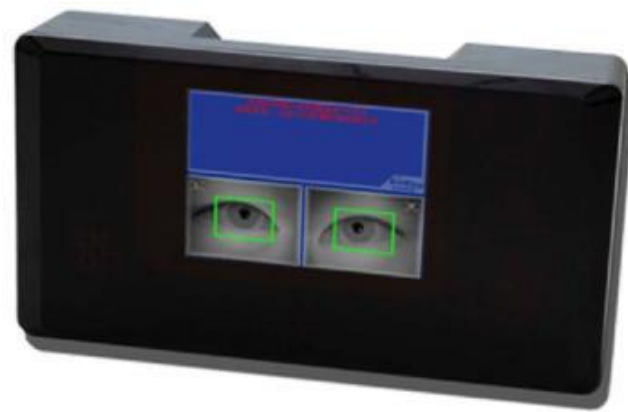

Figure9. CASIA-Iris V4 Thousand imaging device [4] 
The bounding box around the eye shown in the previous figure, tells the human subjects to adjust their positions to get clearer images. This database contains 20,000 images at resolution of $640 * 480$.

The third set, called CASIA-Iris-Syn shown in figure 10, contains 10,000, with resolution of $640 * 480$ synthesized images from CASIA-Iris V1 using the process described in [4]. This process makes the iris texture more realistic and enables it to overcome the noise introduced from motion blur, rotation and deformation.

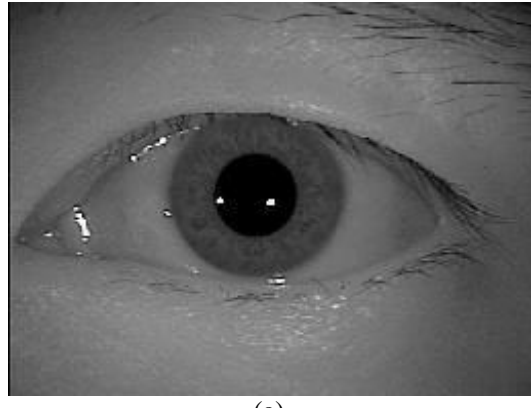

(a)

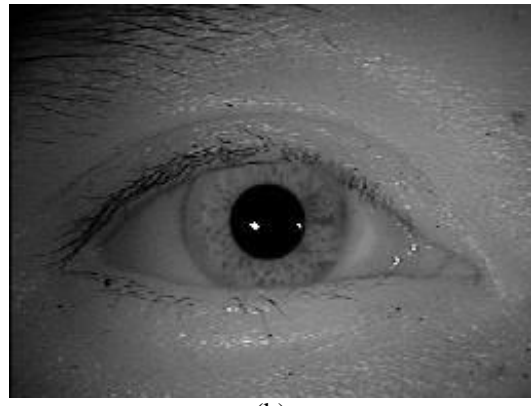

(b)

Fig. 10 Samples from CASIA-Iris V4 Syn [4]

\subsection{BATH IRIS DATABASE}

This database is another example of NIR images databases [5]. This database has been created at the University of Bath by Smart Sensor Limited. Bath iris database consists of 32,000 high quality iris images, captured from 800 mixed ethnic subjects (1600 classes, for left and right eye) with a pixel resolution of $1280 \times 960$. Figure 11 shows samples images from Bath database.

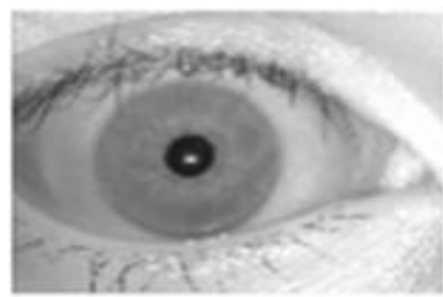

(a)

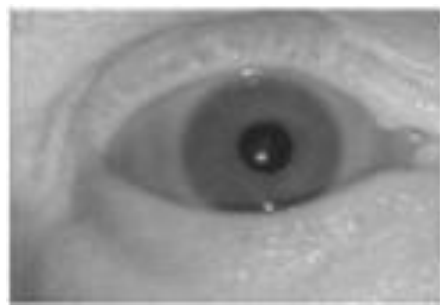

(d)

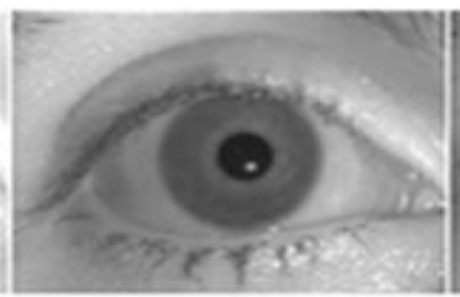

(b)

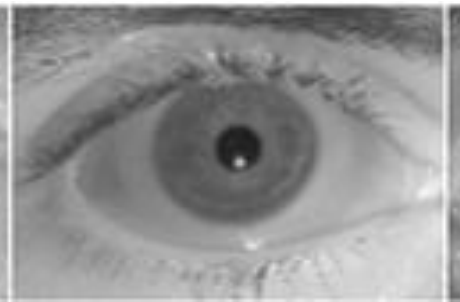

(e)

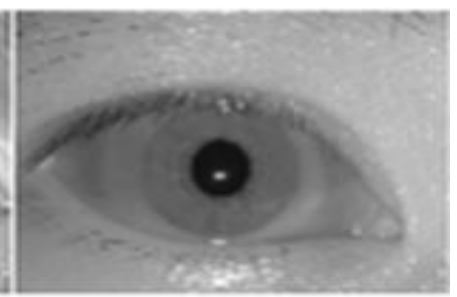

(c)

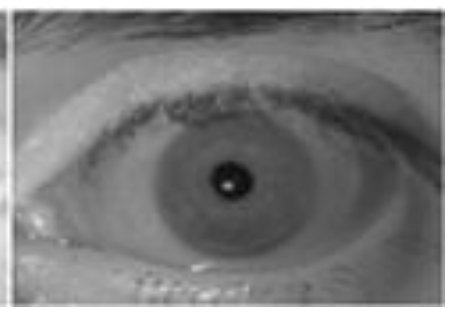

(f)

Figure 11. Samples from Bath iris database [5]

Smart Sensor Limited used the ISG LW-1.3-S-1394 1.3-megapixel camera, placed over an adjustable stand as shown in Figure that have been copied from [6], with array of LED's to capture 200 frames from each subject. Based on the frame quality the best 20 frames are selected to be stored in the database. All the subjects must place their chins on a rest stand over short 
distance from the camera lens, then the camera operator adjust the lens to get the best and the maximum iris texture into focus.

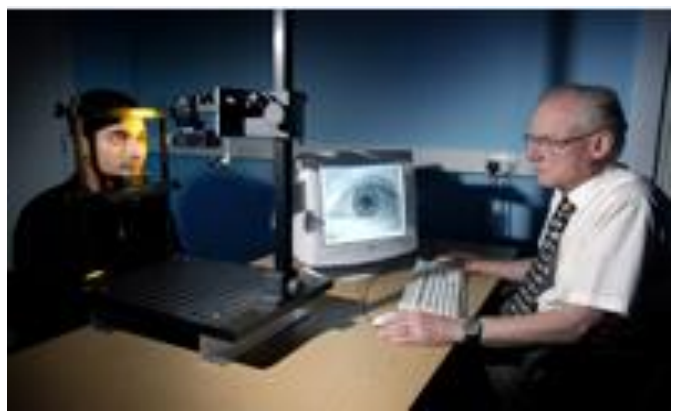

Figure12. Bath database framework [6]

Although the Bath database contains non-ideal images, like images with deviated gaze, occluded with eyelids/eyelashes and focus blur, still the majority of images have homogenous characteristics, and not suitable for the term of unconstrained recognition.

\subsection{MMU IRIS DATABASE}

The Multimedia University has introduced two iris databases [7]. The first one is MMU1 database which comprises of 450 images, captured by a semi-automated camera dedicated for iris capturing (LG IrisAccess 2200) at 7-25 cm distance range, and the second database is MMU2, consisting of 995 images, collected by Panasonic BM-ET100US camera at a distance range of 47$53 \mathrm{~cm}$ from the human subject. The images in MMU database have been collected from 100 volunteers of different ages and nationalities, with each volunteer contributing 5 images from each eye.

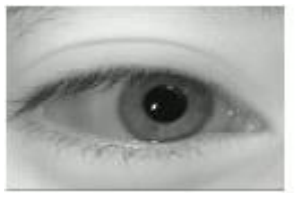

(a)

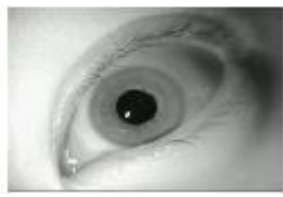

(b)

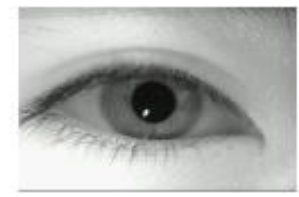

(c)

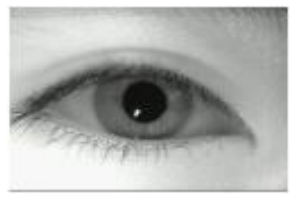

(d)

Figure 13. Samples iris images from MMU database [7]

The iris images in MMU database are homogenous, and have been taken using an NIR light source at a close distance with human subject cooperation, introducing eyelashes obstruction and eye rotation as shown in figure 13.

\subsection{ICE IRIS DATABASE}

In 2005, the National Institute of Standards and Technology (NIST) managed a project under the title of Iris Challenge Evaluation(ICE) [8] to improve and advance the iris recognition technology that supports the current iris recognition systems in the US government. The improvements were made by distributing the problems introduced by exciting iris recognition systems to a potential participant. In 2006, the NIST facilitated the opportunity for competition between participants in a large scale and independent evaluation for testing their algorithms on new larger database using their own frameworks. 
ICE database contains 2954 images, acquired with look and stair, close distance and NIR light source constraints. This database introduces standard noise factors related to poor focus and occlusion of eyelashes Figure 14. Like the previously described databases. The images are not appropriate for the evaluation of unconstrained iris recognition systems.

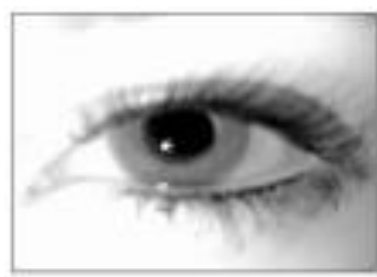

(a)

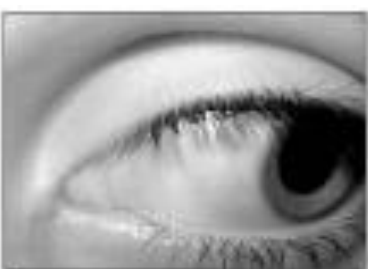

(b)

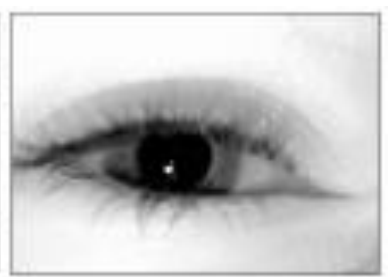

(c)

Figure 14. Samples iris images from ICE database [8]

\subsection{WVU IRIS DATABASE}

WVU iris database was developed at West Virginia University [9]. 380 human subjects contribute three to six images each to produce a database of 1852 iris images, and the acquisition process was performed through a hand-held camera called OKI Iris Pass-H. As shown in Figure 15, the iris images in WVU database have heterogeneous characteristics and they introduce several types of noise factors, such as off-angle, obstructions, poor focus, environment light reflections and rotated images.

The less constrained imaging conditions, makes the WVU iris database a challenging dataset for testing unconstrained iris recognition algorithms, but still the capturing framework uses the NIR light source.

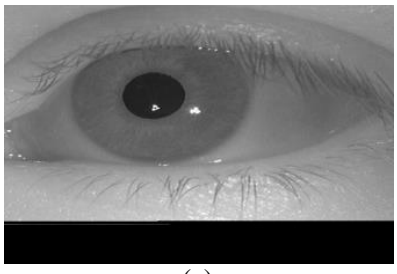

(a)

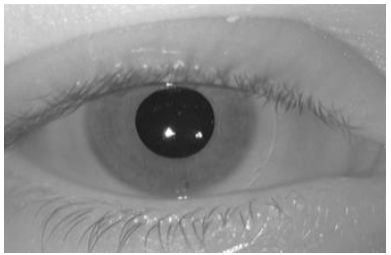

(b)

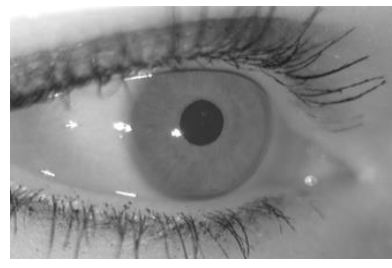

(c)

Figure 15. Samples iris images from WVU database [9]

\subsection{UPOL IRIS DATABASE}

The UPOL (University of Palackeho and Olomouc) [10] is the first database that used imaging framework with a visible wavelength light source. The database comprises of 384 images collected from 64 human subjects, with each subject contributing 6 images ( 3 from left eye and 3 from right eye), and a SONY DXC-950P 3CCD camera connected to TOPCON TRC50IA optical device was used for iris capturing, at resolution of 576×768 RGB 24-bit colour depth Figure 16.

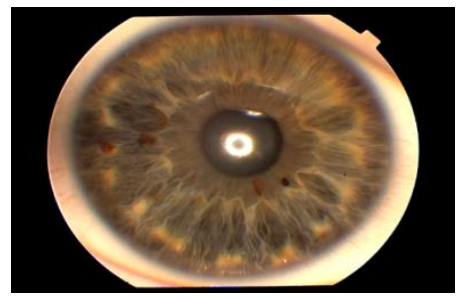

Figure 16. Sample from UPOL iris database [10] 
The images were captured at a close distance (optometric device) with human subject cooperation. Even the visible wavelength light source was placed in a position that does not affect the human iris, and, therefore, the output was extremely noise free images and has homogeneous characteristics, which makes this database unsuitable for testing the unconstrained iris recognition algorithms.

\subsection{UBIRIS V1 IRIS DATABASE}

The reason behind the creation of UBIRIS database [11] is the need of a new noisy iris images dataset, which simulates the less constrained environment in the capturing framework that will be used as a tool for the development of robust recognition proposals.

In contrast to the previously described noise free iris databases apart from WVU database, the UBIRIS database is the noisiest one, which introduces new noise factors caused by (1) motion blur and rotated iris images from moving subjects, (2) poor focus images, (3) obstruction of eyelids and eyelashes, (4) specular and light reflection from visible wavelength light source, and finally (5) closed eye iris images. These noise factors make the UBIRIS database an appropriate set for the developments of unconstrained iris recognition algorithms.

The UBIRIS database, which was created at the University of Beira in 2004, contains 1877 iris images contributed from 241 human subjects, collected through two different sessions with a two weeks' interval, using Nikon E5700v1.0 camera, $71 \mathrm{~mm}$ focal length, 1/30s exposure time. The output image is RGB at $2560 \times 1704$ resolution, 300 dpi vertical and horizontal resolution, with a color depth of 24 bit. The image size was reduced to $300 \times 400$ grey scale to reduce the database size so it can be easily uploaded and downloaded from the web. As shown in figure 17, the first session contains images with minimal noise factors, especially those related to reflection, contrast and luminosity factors. The second session involve more noise factors caused by natural light source, a sample of UBIRISv.1 images is given in figure 18.

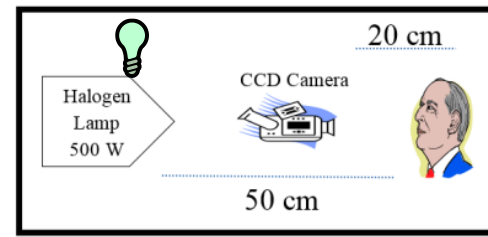

(a)

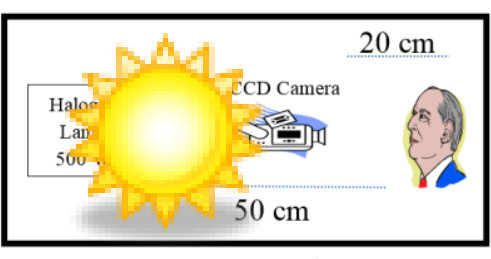

(b)

Figure 17. UBIRIS V1 framework (a) under artificial light source (b) under sun light source [11]

Images in UBIRIS database as stated in Table 1 were manually classified according to three parameters (Focus, Reflection and Visible Iris), and classified as Good, Average and Bad as follows [12]:

Table 1. Image quality in UBIRIS V1 database [12]

\begin{tabular}{|l|l|l|l|}
\hline \multicolumn{1}{|c|}{ Parameter } & Good & Average & Bad \\
\hline \multicolumn{4}{|c|}{ First Session } \\
\hline Reflection & $76.10 \%$ & $16.51 \%$ & $7.39 \%$ \\
\hline Focus & $78.52 \%$ & $19.09 \%$ & $1.99 \%$ \\
\hline
\end{tabular}


The International Journal of Multimedia \& Its Applications (IJMA) Vol.9, No.2, April 2017

\begin{tabular}{|l|l|l|l|}
\hline Visible Iris & $43.65 \%$ & $52.86 \%$ & $3.49 \%$ \\
\hline \multicolumn{4}{|c|}{ Second Session } \\
\hline Reflection & $69.70 \%$ & $19.39 \%$ & $10.91 \%$ \\
\hline Focus & $22.27 \%$ & $69.09 \%$ & $8.64 \%$ \\
\hline Visible Iris & $24.09 \%$ & $38.64 \%$ & $37.27 \%$ \\
\hline
\end{tabular}

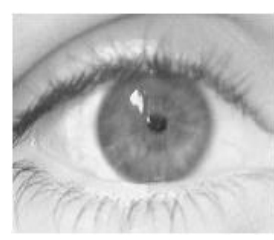

(a)

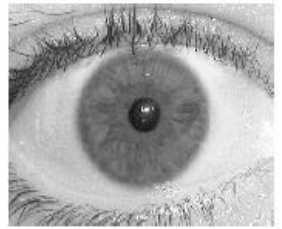

(e)

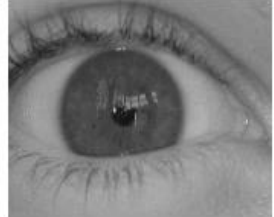

(b)

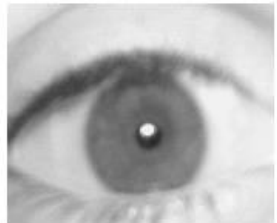

(f)

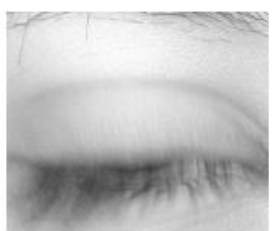

(c)

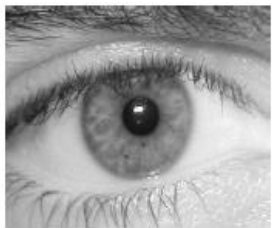

(g)

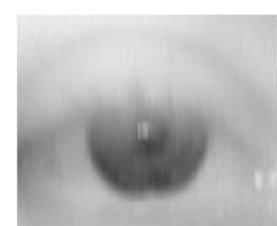

(d)

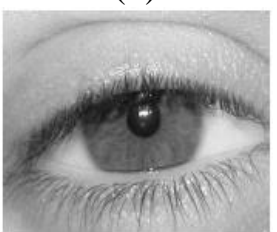

(h)

Figure18. Samples from UBIRIS V1 iris database [11]

\subsection{UBIRIS V2 DATABASE}

In 2010, the University of Beira introduced another version of UBIRIS database called UBIRIS.v2 [13] iris database, which is a multisession iris database that contains images captured at a distance of 4 to 8 meters, on the move and lit with a visible wavelength light source. The reason behind the creation of UBIRIS.v2 database is to constitute a new iris dataset collected under a natural or artificial visible wavelength light source, with characteristics far away from ideal imaging framework.

The Canon EOS 5D was used, with 1/197 shutter speed, 400mm focal length, 1/200s exposure time and ISO-1600 speed. The resultant image is $800 \times 600$, with $72 \mathrm{dpi}$ vertical/horizontal resolution and 24-bit color depth, stored in tiff file format.

Unlike UBIRIS.v1, images in UBIRIS.v2 were captured over a distance of 4 to 8 meters. The subjects were asked to walk in average speed slower than normal towards the acquisition camera. Three images per meter were captured while the human subject was looking at preplaced lateral signs. That obliged them to rotate their heads.

There were 261 volunteers participating with $522(261 \times 2$ left and right $)$ irises, producing 11,102 images. Like UBIRIS.v1, the images have been captured in two different sessions, with a twoweek interval. 60 percent of the volunteers participated in both sessions and 40 percent participated either in the first or second sessions.

Images in UBIRIS.v2 database have heterogeneous characteristics, with 14 different types of noise, "((1) Off-angle iris image, (2) Poorly focused iris image, (3) Rotated iris image, (4) Motion-blurred iris image, (5) Iris obstructions due to eyelids, (6) Iris obstructions due to 
eyelashes, (7) Iris obstructions due to glasses, (8) Iris obstructions due to contact lenses, (9) Iris obstructions due to hair, (10) Iris imaging in poor lighting conditions, (11) Iris with specular reflections, (11) Iris with lighting reflections, (12) Partially captured iris, (13) Dark iris, and (14) Out-of-iris image)" [13], see Fig. 19. This heterogeneity makes this database an appropriate set for evaluation of unconstrained iris recognition algorithms.

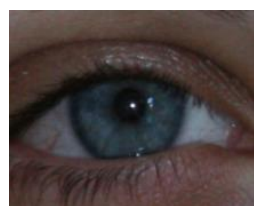

(a)

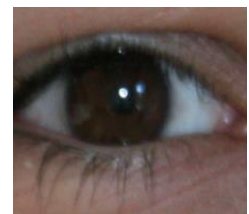

(f)

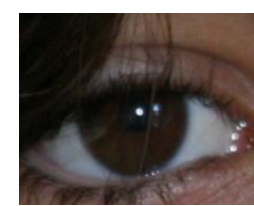

(b)

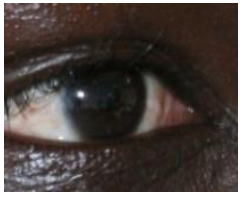

(g)

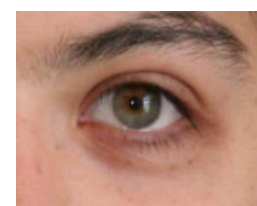

(c)

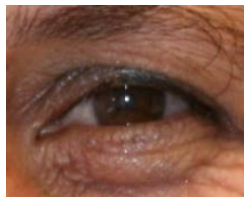

(h)

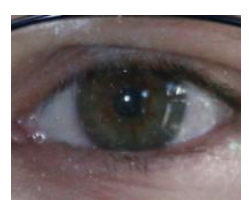

(d)

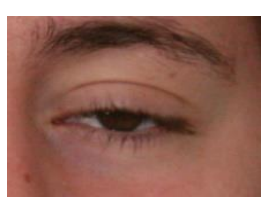

(i)

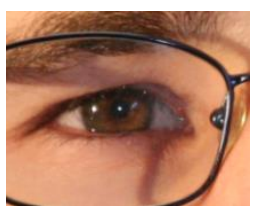

(e)

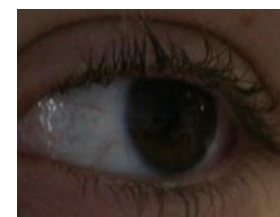

(j)

Figure19. Samples from UBIRIS V2 iris database [13]

\section{FRGC (FACE RECOGNition GRAND ChalLenge)}

The reason behind the creation of FRGC database [14] was to support and advance the efforts in the U.S. Government for developing a robust face recognition technology, the database was opened for researchers and academic institutions for development from 2004 to 2006.

The FRGC database was designed for face recognition researchers. Nevertheless, recent advances in iris recognition like in [15] used images from FRGC database to test their method against severe unconstrained conditions. FRGC consists of 50,000 class divided into training and validation partitions captured from 4,003 human subjects. Each class consists of four controlled still images captured in a studio, two uncontrolled images and one $3 \mathrm{~d}$ image. All images were captured under different lighting conditions.

In Fig. 20. The first two images from the left are images captured in controlled environment, and the two images on the right are images captured in unconstrained environment.

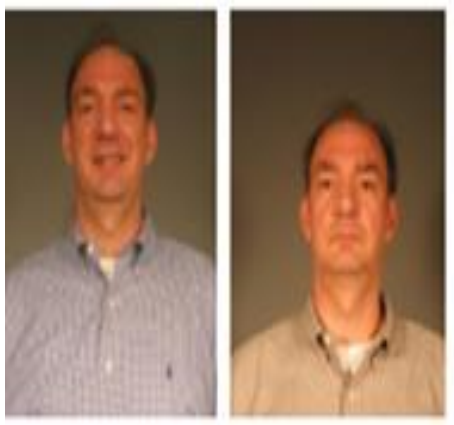

(a)

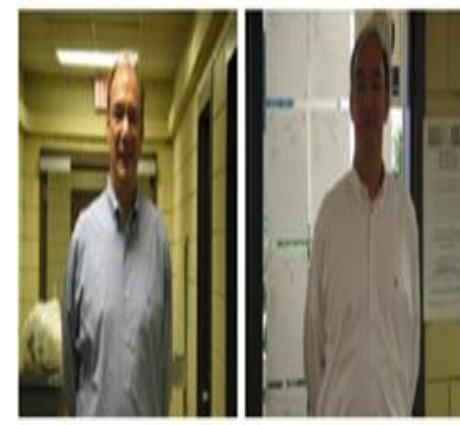

(b)

Figure 20. Samples from FRGC database 
The International Journal of Multimedia \& Its Applications (IJMA) Vol.9, No.2, April 2017

\section{COMPARISON OF Iris DATABaSES}

For a comprehensive comparison, the iris databases were compared according to five criteria's (database size, light source, imaging distance and the noise factors). Table 2 and Table 3 summarize the free available iris databases and their characteristics.

Table 2. Comparison of Free iris database [13]

\begin{tabular}{|c|c|c|c|c|c|}
\hline $\begin{array}{l}\text { Database } \\
\text { name }\end{array}$ & $\begin{array}{l}\text { Dutubust } \\
\text { sint }\end{array}$ & $\begin{array}{l}\text { Light } \\
\text { wawe } \\
\text { leaght }\end{array}$ & $\begin{array}{l}\text { Vurving } \\
\text { ditunce }\end{array}$ & Camera & Sample image \\
\hline CASLA $\mathrm{rl}$ & 786 & $\mathrm{NL}$ & No & CASIA caman & \\
\hline CASLA 12 & 2255 & $\mathrm{NR}$ & No & CASIA caman & \\
\hline CASLA $¥$ & 22,051 & $\mathrm{NR}$ & No & oxI inipus h & \\
\hline CASLA T4 & 2,576 & $\mathrm{NR}$ & Yo: & IEEMB-100 tual caman & \\
\hline Bath & 16,000 & $\mathrm{NR}$ & No & ISGLW 1.351394 & \\
\hline MMU 1 & 450 & $\mathrm{NR}$ & No & LG EOU 200 & \\
\hline MMU 2 & 995 & $\mathrm{NR}$ & No & Punouic EM ET 100 US & \\
\hline ICE 1 & 2900 & $\mathrm{NR}$ & No & LG EOU noo & \\
\hline ICE 2 & 75,000 & $\mathrm{NL}$ & No & LG EOU 200 & \\
\hline wvo & 3099 & $\mathrm{NL}$ & No & OKI iñ-pas h & \\
\hline UPOL & 334 & Vivibla & No & $\begin{array}{l}\text { Sory DXC 950P } 3000 \text { with } \\
\text { TOPOON TRCSOLA }\end{array}$ & \\
\hline $\begin{array}{l}\text { UBIRIS } \\
\mathrm{vl}\end{array}$ & 1877 & Vivitla & No & NIKON E 5700 & \\
\hline $\begin{array}{l}\text { LBIRIS } \\
\mathrm{v} 2\end{array}$ & 11,357 & Vivitla & Yas & Coman EOS 5D & \\
\hline FRGC & 5,000 & Viaibla & Yw & Minolta Viria 900910 & \\
\hline
\end{tabular}




\begin{tabular}{|c|c|c|c|c|c|c|c|c|c|c|c|c|c|c|}
\hline \multirow[b]{2}{*}{ Database } & \multicolumn{14}{|c|}{ Noise } \\
\hline & 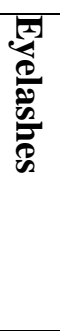 & 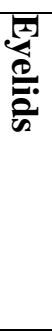 & 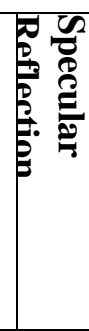 & 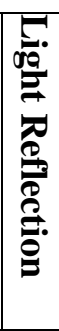 & 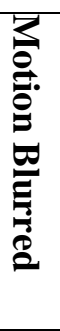 & 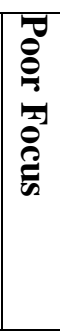 & 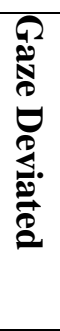 & 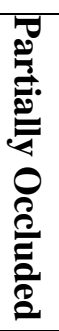 & 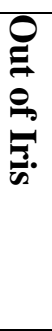 & 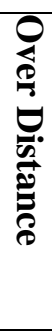 & 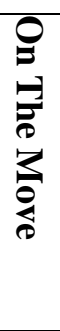 & $\begin{array}{l}\overline{0} \\
\stackrel{0}{2} \\
\stackrel{\overrightarrow{2}}{2}\end{array}$ & 足 & 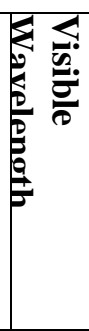 \\
\hline CASIA 1 & $\sqrt{ }$ & $\sqrt{ }$ & - & - & - & - & - & - & - & - & - & - & - & - \\
\hline CASIA 2 & $\sqrt{ }$ & $\sqrt{ }$ & - & - & - & - & - & - & - & - & - & - & - & - \\
\hline CASIA 3 & $\sqrt{ }$ & $\sqrt{ }$ & - & - & - & - & - & - & - & - & - & - & - & - \\
\hline CASIA 4 & $\sqrt{ }$ & $\sqrt{ }$ & - & - & $\sqrt{ }$ & $\sqrt{ }$ & $\sqrt{ }$ & $\sqrt{ }$ & - & $\sqrt{ }$ & $\sqrt{ }$ & $\sqrt{ }$ & - & - \\
\hline BATH & $\sqrt{ }$ & $\sqrt{ }$ & - & - & - & - & $\sqrt{ }$ & - & - & - & - & $\sqrt{ }$ & - & - \\
\hline MMU 1 & $\sqrt{ }$ & $\sqrt{ }$ & - & - & - & - & - & - & - & - & - & - & - & - \\
\hline MMU 2 & $\sqrt{ }$ & $\sqrt{ }$ & - & - & - & - & - & - & - & - & - & - & - & - \\
\hline ICE 1 & $\sqrt{ }$ & $\sqrt{ }$ & - & - & - & - & $\sqrt{ }$ & - & - & - & - & $\sqrt{ }$ & - & - \\
\hline ICE 2 & $\sqrt{ }$ & $\sqrt{ }$ & - & - & - & - & $\sqrt{ }$ & $\sqrt{ }$ & $\sqrt{ }$ & - & - & $\sqrt{ }$ & - & - \\
\hline WVU & $\sqrt{ }$ & $\sqrt{ }$ & - & - & $\sqrt{ }$ & $\sqrt{ }$ & $\sqrt{ }$ & $\sqrt{ }$ & - & - & $\sqrt{ }$ & $\sqrt{ }$ & - & - \\
\hline UPOL & - & - & - & - & - & - & - & - & - & - & - & - & - & $\sqrt{ }$ \\
\hline UBIRIS.v1 & $\sqrt{ }$ & $\sqrt{ }$ & $\sqrt{ }$ & $\sqrt{ }$ & $\sqrt{ }$ & $\sqrt{ }$ & $\sqrt{ }$ & $\sqrt{ }$ & $\sqrt{ }$ & - & - & $\sqrt{ }$ & $\sqrt{ }$ & $\sqrt{ }$ \\
\hline UBIRIS.v2 & $\sqrt{ }$ & $\sqrt{ }$ & $\sqrt{ }$ & $\sqrt{ }$ & $\sqrt{ }$ & $\sqrt{ }$ & $\sqrt{ }$ & $\sqrt{ }$ & $\sqrt{ }$ & $\sqrt{ }$ & $\sqrt{ }$ & $\sqrt{ }$ & $\sqrt{ }$ & $\sqrt{ }$ \\
\hline FRGC & $\sqrt{ }$ & $\sqrt{ }$ & $\sqrt{ }$ & $\sqrt{ }$ & $\sqrt{ }$ & $\sqrt{ }$ & $\sqrt{ }$ & $\sqrt{ }$ & $\sqrt{ }$ & $\sqrt{ }$ & $\sqrt{ }$ & $\sqrt{ }$ & $\sqrt{ }$ & $\sqrt{ }$ \\
\hline
\end{tabular}

\section{APPLICATIONS OF IRIS RECOGNITION}

Iris recognition as a biometric identification has enormous potential for security in many fields. Recently iris recognition has been applied in airline travel, border security check (UAE Dubai, UK Heathrow and USA Dulles airports), bank customer ID (Cairo Amman Bank). Moreover, the iris recognition is used for identifying immigrants, access to highly restricted areas (US Military), police evidence room (USA) and ATM (Bank United in Houston) [16].

\section{Conclusion}

This research summarizes all free available iris databases, these databases are categorized in two types, and the first category contains the noise free iris databases that have been captured under NIR light source (CASIA, BATH, MMU, ICE and WVU). While the noisy iris databases that 
have been captured under visible wavelength light source (UPOL, UBIRISv.1 and UBIRISv.2) fall in the second category. In this survey an attempt has been made to present the commonly used iris databases which provides a platform for the development of new robust proposals in the area of iris recognition. In the future work, we are planning to improve the segmentation accuracy for UBIRIS V.2 database, the heterogeneity of the characteristics of UBIRIS V.2 database makes it an appropriate set for evaluation of unconstrained iris recognition algorithms.

\section{REFERENCES}

[1] J. G. Daugman, "High confidence visual recognition of persons by a test of statistical independence," Transactions on Pattern Analysis and Machine Intelligence, IEEE, vol. 15, pp. 1148-1161, 1993.

[2] J. Daugman, "How Iris Recognition Works," IEEE Transactions on Circuits and Systems for Video Technology, vol. 14, pp. 21-30, 2004.

[3] J. Daugman, "The importance of being random: statistical principles of iris recognition," Pattern Recognition Society, pp. 279-291, 2002.

[4] BIT. CASIA Iris Image Database [Online]. Available: http://biometrics.idealtest.org/. Last Accessed (Dec 2016).

[5] Smart-Sensor-Ltd. BATH Iris Database [Online]. Available: http://www.smartsensors.co.uk/products/iris-database/. Last Accessed (Dec 2016).

[6] SmartSensores. Available: [Online] http://www.smartsensors.co.uk/products/iris-database/. Last Accessed (Dec 2016).

[7] Multimedia-University. MMU Database [Online]. Available: http://pesona.mmu.edu.my/ ccteo/. Last Accessed (Dec 2016).

[8] National-Institute-of-Standard-and-Technology. Iris Challenge Evaluation Database [Online]. Available: http://www.nist.gov/itt/iad/ig/ice.cfm. Last Accessed (Dec 2016).

[9] West-Virginia-University. WVU Iris Database [Online]. Available: http://www.csee.wvu.edu/ xinl/demo/nonideal_iris.html. Last Accessed (Dec 2016).

[10] University-of-Palackeho-and-Olomouc. UPOL Iris Database [Online]. Available: http://phoenix.inf.upol.cz/iris/. Last Accessed (Dec 2016).

[11] H. Proença and L. Alex, "UBIRIS: A noisy iris image database," in Proceedings of the 13th International Conference on Image Analysis and Processing - ICIAP, 2005.

[12] H. Proença and L. A. Alexandre, "Toward noncooperative iris recognition: a classification approach using multiple signatures," Transactions on Pattern Analysis and Machine Intelligence, vol. 29, pp. 607-12, Apr 2007.

[13] H. Proença, S. Filipe, R. Santos, J. Oliveira, and L. A. Alexandre, "The UBIRIS.v2: a database of visible wavelength iris images captured on-the-move and at-a-distance," IEEE Transaction Pattern Analysis and Machine Intelligence, vol. 32, pp. 1529-35, Aug 2010.

[14] UK. brp-information-leaflet. [Online] https://www.gov.uk/government/uploads/system/uploads/attachment_data/file/261497/brpinformation-leaflet.pdf. Last Accessed (Dec 2016).

[15] S. Hosseini and S. Mohammad, "Review Banking on Biometric in the World's Banks and Introducing a Biometric Model for Iran's Banking System," Basic and Applied Scientific Research, vol. 2, pp. 9152-9160, 2012.

[16] SANS Institute InfoSec Reading Room. Available [Online]: http://www.sans.org/readingroom/whitepapers/authentication/iris-recognition- technology-improvedauthentication-132. Last Accessed (Dec 2016).

\section{Authors}

Mustafa M Alrifaee was born in Zarqa Jordan, in 1978. He received the B.Sc. and M.Sc. degrees in information technology from the University of Sindh, Hyder-Abad, Pakistan, in 2000 and 2001, respectively. He received Ph.D. degree in Multimedia from the Faculty of Technology, University of De Montfort, Leicester, UK, in 2015.In 2002, he joined the Department of Computer Information System, University of Al-Zaytoonah, as a Lecturer, and in 2015 became an Assistant Professor. His current research interests include pattern

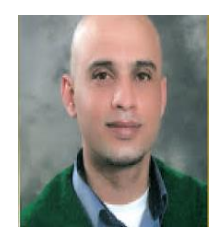


recognition, signal processing and software testing.Dr. Mustafa is a member in the Department of Multimedia System, University of Al-Zaytoonah, Amman, Jordan.

Mohammad M. Abdallah was born in Kuwait 1985. He received his Ph.D. in Software Engineering from Durham University, UK, in 2012; his Master's degree in software engineering from Bradford University, UK, in 2008; and his Bachelor's degree in computer science from Al-Zaytoonah University, Amman, Jordan, in 2007. He has been a member of the Faculty of Science and Information Technology at Al-Zaytoonah University since 2012, where he held different positions including the Chair of Software Engineering Department, and now, he is the Chair of Computer Science Department. His current research interested is Software Measurement, Software Quality and Program analysis. Dr. Abdallah is a professional member in ACM.

Basem G. Al Okush, was born in Jordan 1980, Obtain master degree in Computer Science from New York Institute of Technology (NYiT), Amman, Jordan in 2004, and Bachelor of Computer Science from Al - Zytoonah University of Jordan, Amman, Jordan in 2002. He held different positions; Lecturer in Al-Zaytoonah University since 2008, before that he was a teacher assistant in Al-Zaytoonah since 2002. His research interest in Program analysis and software testing.

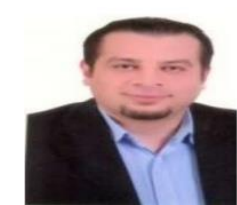

